\title{
Silica Nanoparticles Sensitize Human Multiple Myeloma Cells to Snake (Walterinnesia aegyptia) Venom-Induced Apoptosis and Growth Arrest
}

\author{
Douaa Sayed, ${ }^{1}$ Mohamed K. Al-Sadoon, ${ }^{2}$ and Gamal Badr ${ }^{3,4}$ \\ ${ }^{1}$ Clinical Pathology Department, South Egypt Cancer Institute, Assiut University, Assiut 171515, Egypt \\ ${ }^{2}$ Zoology Department, College of Science, King Saud University, Riyadh 11451, Saudi Arabia \\ ${ }^{3}$ Princess Johara Alibrahim Center for Cancer Research, Prostate Cancer Research Chair, College of Medicine, \\ King Saud University, P.O. Box 7805, Riyadh 11472, Saudi Arabia \\ ${ }^{4}$ Zoology Department, Faculty of Science, Assiut University, Assiut 71516, Egypt
}

Correspondence should be addressed to Gamal Badr, badr73@yahoo.com

Received 26 September 2012; Revised 2 November 2012; Accepted 2 November 2012

Academic Editor: Zhao Zhong Chong

Copyright (๑) 2012 Douaa Sayed et al. This is an open access article distributed under the Creative Commons Attribution License, which permits unrestricted use, distribution, and reproduction in any medium, provided the original work is properly cited.

Background. Multiple myeloma (MM), an almost incurable disease, is the second most common blood cancer. Initial chemotherapeutic treatment could be successful; however, resistance development urges the use of higher toxic doses accompanied by hematopoietic stem cell transplantation. The establishment of more effective treatments that can overcome or circumvent chemoresistance has become a priority. We recently demonstrated that venom extracted from Walterinnesia aegyptia (WEV) either alone or in combination with silica nanoparticles (WEV+NPs) mediated the growth arrest and apoptosis of prostate cancer cells. In the present study, we evaluated the impact of WEV alone and WEV+NP on proliferation and apoptosis of MM cells. Methods. The impacts of WEV alone and WEV+NP were monitored in MM cells from 70 diagnosed patients. The influences of WEV and WEV+NP were assessed with flow cytometry analysis. Results. WEV alone and WEV+NP decreased the viability of MM cells. Using a CFSE proliferation assay, we found that WEV+NP strongly inhibited MM cell proliferation. Furthermore, analysis of the cell cycle using the propidium iodide (PI) staining method indicated that WEV+NP strongly altered the cell cycle of MM cells and enhanced the induction of apoptosis. Conclusions. Our data reveal the biological effects of WEV and WEV+NP on MM cells that enable these compounds to function as effective treatments for MM.

\section{Background}

Multiple myeloma, an almost incurable disease, is the second most common blood cancer. It is characterized by the presence of malignant plasma cells that are predominantly located in the bone marrow. The treatment of myeloma is mainly directed at the management of complications and reduction of malignant cell masses. Initial chemotherapeutic treatment could be successful; however, resistance development urges the use of higher toxic doses accompanied by hematopoietic stem cell transplantation [1].

Therefore, to improve outcomes and extend the length of survival, the establishment of more effective treatments that can overcome the complications or circumvent chemoresistance has become a priority [2].
Natural toxins are recognized as sources for drugs against several human ailments, including cancers. In particular, a nontoxic dose of snake venom was shown to both reduce the solid tumor size of ovarian cancers and block the process of angiogenesis [3]. The search for new anticancer agents remains necessary to increase the number of available options and identify less toxic and more effective drugs. Snake venom is a complex mixture of many substances, including toxins, enzymes, growth factors, activators, and inhibitors, with a wide spectrum of biological activities. Our recent studies have demonstrated the antitumor potential of snake venom from Walterinnesia aegyptia (WEV) on the human breast carcinoma cell line MDA-MB-231 and have shown its effect on normal murine peripheral blood mononuclear cells (PBMCs) [4]. In addition, other data have 
indicated that a nanogram concentration range of snake venom toxin from Vipera lebetina turanica inhibits hormonerefractory human prostate cancer cell growth; this effect is related to the NF- $\kappa \mathrm{B}$ signal-mediated induction of apoptosis [5].

Nanoparticles carrying chemical therapeutics have shown great promise in treating cancer patients. When loaded with anticancer agents, nanoparticles can successfully increase drug concentrations in cancer tissues and act at the cellular level to enhance antitumor efficacy. The nanoparticles can be endocytosed and/or phagocytosed by cells, which results in the internalization of the encapsulated drug [6]. Therefore, in the present study, we investigated the effects of WEV alone and in combination with silica nanoparticles (WEV+NPs) on the proliferation and apoptosis of MM cells.

\section{Materials and Methods}

2.1. Samples. Bone marrow (BM) aspirate samples from 70 patients with MM were obtained from the South Egypt Cancer Institute and the Assiut University Hospital of Assiut University in Egypt, and approval was obtained from the institutional review board for these studies.

Bone marrow mononuclear cells were examined from heparinized BM aspirate drawn from MM patients (by monitoring the cell-surface expression of CD138) and a viability of $>90 \%$ (by the Trypan blue exclusion method).

\subsection{Preparation of W. aegyptia Venom. W. aegyptia snakes} were collected from the central region of Saudi Arabia and kept in a serpentarium in the Zoology Department, College of Science, King Saud University. The snakes were warmed daily using a 100-watt lamp for nine hours, and water was always available. The snakes were fed purposebred mice every 10 to 14 days. The venom was milked from adult snakes, lyophilized, and reconstituted in $1 \mathrm{X}$ phosphatebuffered saline (PBS) prior to use.

\subsection{Preparing the Combination of W. aegyptia Venom and} Silica Nanoparticles. The combination of silica nanoparticles and snake venom was prepared at King Abdullah Institute for Nanotechnology, King Saud University. A double mesoporous core-shell silica nanosphere was formed around a silica core by using an anionic surfactant; the solid silica core was thus turned into a mesoporous one. First, for the synthesis of the solid silica core, $0.875 \mathrm{~mL}$ aqueous ammonia was added to a solution that contained $18 \mathrm{~mL}$ ethanol and $2.6 \mathrm{~mL}$ deionized water; then, $1.5 \mathrm{~mL}$ tetraethyl orthosilicate (TEOS) was added while the solution was vigorously stirred. The resulting mixture was heated at $30^{\circ} \mathrm{C}$ for $60 \mathrm{~min}$, and then, the silica precipitate was collected by centrifugation and washed three times with water. Second, to synthesize the mesoporous core-shell nanosphere, silica $\left(\mathrm{SiO}_{2}\right)$ particles were dispersed using an anionic surfactant in $15 \mathrm{~mL} \mathrm{H}_{2} \mathrm{O}$ and ultrasonicated for $10 \mathrm{~min}$. To suppress the agglomeration of the silica cores, $1 \mathrm{~g} / \mathrm{L}$ polyvinylpyrrolidone was added with constant stirring for $60 \mathrm{~min}$. Next, $0.1 \mathrm{~mL}$ 3-aminopropyltrimethoxysilane (APMS), $0.2933 \mathrm{~g}$ (1 mmol) $\mathrm{N}$-lauroylsarcosine sodium (Sar-Na), and $1.5 \mathrm{~mL}$ TEOS were added to the reaction mixture with subsequent stirring at $50^{\circ} \mathrm{C}$ for $2 \mathrm{~h}$. The final solid was recovered by centrifugation, washed with deionized water, and dried in an oven at $60^{\circ} \mathrm{C}$ for 12 hours. Template removal was performed using a heat treatment in an air stream at $550^{\circ} \mathrm{C}$ for 6 hours. The resulting molar ratio was TEOS: $\mathrm{H}_{2} \mathrm{O}$ :APMS: Sar$\mathrm{Na}: \mathrm{HCl}: \mathrm{PVP}=1: 331.6: 0.08: 0.14: 0.06: 5 \times 10^{-3}$. After synthesizing the nanoparticles, $25 \mathrm{mg}$ mesoporous silica nanoparticles were added to a solution of $50 \mathrm{mg} / \mathrm{mL}$ venom in $0.5 \mathrm{~mL}$ water. The suspension was stirred for 2 hours, and the evaporation of water was prevented. The mesoporous silica nanoparticles loaded with venom were recovered using high-speed centrifugation and then dried in a vacuum oven at $60^{\circ} \mathrm{C}$. Transmission electron microscopy (TEM) was performed using a JEOL JSM-2100F electron microscope (Japan) operated at $200 \mathrm{kV}$. Nitrogen sorption isotherms were measured at $77 \mathrm{~K}$ with a Quantachrome NOVA 4200 analyzer (USA). Prior to taking measurements, the samples were degassed in a vacuum at $200^{\circ} \mathrm{C}$ for at least 18 hours. The Brunauer-Emmett-Teller (BET) method was utilized to calculate the specific surface areas (SBET) using adsorption data in a relative pressure range from 0.05 to 0.35 . By using the Barrett-Joyner-Halenda (BJH) model, the pore volumes and pore size distributions were derived from the adsorption branches of the isotherms, and the total pore volumes $\left(V_{t}\right)$ were estimated from the adsorbed amount at a relative pressure $\left(P / P^{0}\right)$ of 0.992 .

To determine the effect of the reaction time on the interaction between the silica nanoparticles and the snake venom, various reaction times for the synthesis of the solid silica cores were tested; the formation of double mesoporous coreshell silica spheres resulted as shown in Figure 1. At both the 1- and 6-h reaction times, a homogenous mesoporous silica shell was observed. However, at the 1-h reaction time, the silica core seemed to be quite dense. Extending the reaction time for the synthesis of the solid silica cores up to $6 \mathrm{~h}$ resulted in clear and pronounced mesopores that were radially oriented; these mesopores were anchored within the core and extended from the core center up to the shell as shown in Figure 1(b). The decay of core contrast as the reaction time increased from $1 \mathrm{~h}$ to $6 \mathrm{~h}$ suggested the anchoring of more mesopores and a loss of core density as the reaction time increased; this was also indicated by the higher pore volume observed with the 6 -h reaction time. In contrast, the shell thickness did not change significantly with different reaction times; the thickness remained approximately $50 \mathrm{~nm}$. The particle size distribution shows that both samples generated a particle size of approximately $300 \mathrm{~nm}$. However, the 6-h reaction sample had a narrower distribution than the 1-h sample as shown in Figure 2. Figure 3 shows the $\mathrm{N}_{2}$ adsorption-desorption isotherms generated by different surfactant reaction times; these isotherms exhibit a type IV shape, which is characteristic of mesoporous materials. The total pore volume was measured to be 0.342 and $0.416 \mathrm{cc} / \mathrm{g}$ at the 1- and 6-h reaction times, respectively. The observation of a triangular hysteresis loop between the adsorption and desorption branches can be attributed to the coexistence of 


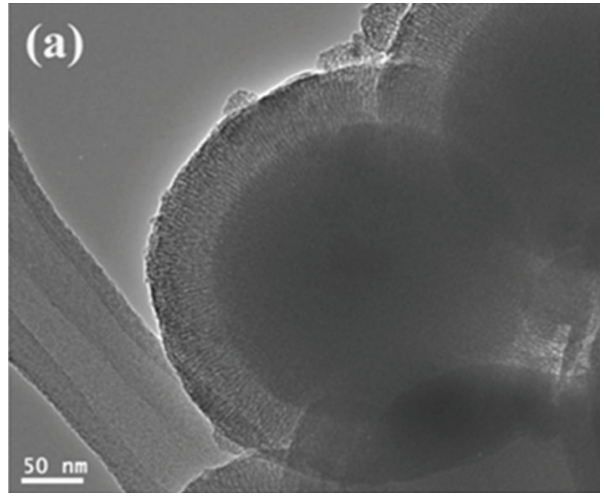

(a)

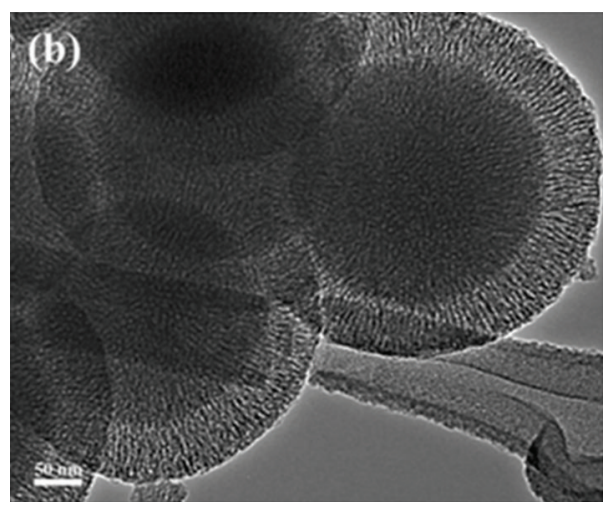

(b)

FIGURE 1: TEM images of calcined double mesoporous core-shell silica nanospheres prepared from silica cores formed at different reaction time of (a) 1, (b) $6 \mathrm{~h}$.

the hexagonal and lamellar phases. The BET surface area was 304.08 and $440.73 \mathrm{~m}^{2} / \mathrm{g}$ at the 1 - and 6 -h reaction times, respectively. It is clear that the textural characteristics of the mesoporous core-shell structure were enhanced by increasing the reaction time.

2.4. Monoclonal Antibodies. For immunostaining and analysis by fluorescence-activated cell sorting (FACS), we used Allophycocyanin (APC), Phycoerythrin (PE), and Fluorescein isothiocyanate- (FITC-), conjugated mouse monoclonal antibodies (mAbs) against human CD3, CD138 (BD PharMingen, SanDiego, CA, USA), and Annexin V (IQ products, Netherlands) and the corresponding mouse isotype controls (all from BD PharMingen, SanDiego, CA, USA). CD3 is specific for lymphocytes, and CD138 is specific for MM cells. All antibodies were used at concentrations titrated for optimal staining. The staining of monoclonal antibodies was carried out using a standard protocol.

2.5. Flow Cytometry. Flow cytometry was performed with the FACSCalibur system (BD, San Jose, CA, USA). All fluorocytometric data were subsequently analysed and displayed with CELLQUEST software (BD, San Jose, CA, USA). Each analysis included measurements from a minimum of 20000 cells.

2.6. CFSE Proliferation Assay. BM cells washed twice in PBS and stained with $0.63 \mu \mathrm{M}$ carboxyfluorescein diacetate succinimidyl ester (CFSE) (Molecular Probes, Eugene, OR, USA) for $8 \mathrm{~min}$ at room temperature. Residual CFSE was removed by washing three times in PBS. CFSE-labeled cells were then seeded in 6-well plates, treated with NP, WEV, or WEV+NP or left untreated (0) and grown for 4 days in RPMI-cell culture medium. The cells then were stained with the monoclonal antibodies. The CFSE fluorescence intensity was measured by flow cytometry. MM cells were gated manually and created (R1) basis on positive CD138. Expression of cell markers was assessed by looking at the relative number of positive cells. Isotype control antibodies were used to separate positive and negative cells.

2.7. Flow Cytometric Analysis of Apoptosis and Cell Cycle Analysis. After treatment with NP, WEV, or WEV+NP, MM cells were fixed and permeabilized with 70\% ice-cold ethanol for at least $1 \mathrm{~h}$ and then washed twice in PBS. DNA was stained by incubating the cells at $37^{\circ} \mathrm{C}$ for $1 \mathrm{~h}$ in $40 \mu \mathrm{g} / \mathrm{mL}$ propidium iodide and $100 \mu \mathrm{g} / \mathrm{mL}$ DNase-free RNase in PBS. As the fluorescence area (FL2-A) is the main parameter in the cell cycle analysis, a histogram plot of FL2-A serves as a cell cycle graph using Modfit LT 3.0 software (BD, San Jose, CA, USA) were analysed.

Dead cells were identified using the Trypan blue exclusion test. Also MM cells were reanalyzed for expression of annexin $\mathrm{V}$ and both annexin V and PI to identify early and late apoptosis, respectively.

2.8. Caspases Activity. Caspases 3, 8, and 9 activities were evaluated using a fluorometric protease assay kit (MBL, Aichi, Japan), according to the manufacturer's instructions.

2.9. ROS Measurement. Reactive oxygen species (ROS) levels were determined using 2,7-dichlorodihydrofluorescein diacetate (H2DCF-DA) (Beyotime Institute of Biotechnology, Haimen, China $)$. MM cells $(1 \times 106)$ were directly treated with $10 \mathrm{lM} \mathrm{H} 2 \mathrm{DCF}-\mathrm{DA}$ dissolved in $1 \mathrm{~mL} \mathrm{PBS}$ at $37^{\circ} \mathrm{C}$ for $20 \mathrm{~min}$. The fluorescence intensity was monitored using an excitation wavelength of $488 \mathrm{~nm}$ and an emission wavelength of $530 \mathrm{~nm}$.

2.10. Hydroperoxide Levels. Levels of hydroperoxide were evaluated using the free radical analytical system (FRAS 2, Iram, Parma, Italy). This test is a colorimetric assay that takes advantage of the ability of hydroperoxide to generate free radicals after reacting with some transitional metals.

2.11. Statistical Analysis. The data were first tested for normality using the Anderson-Darling test and then evaluated 


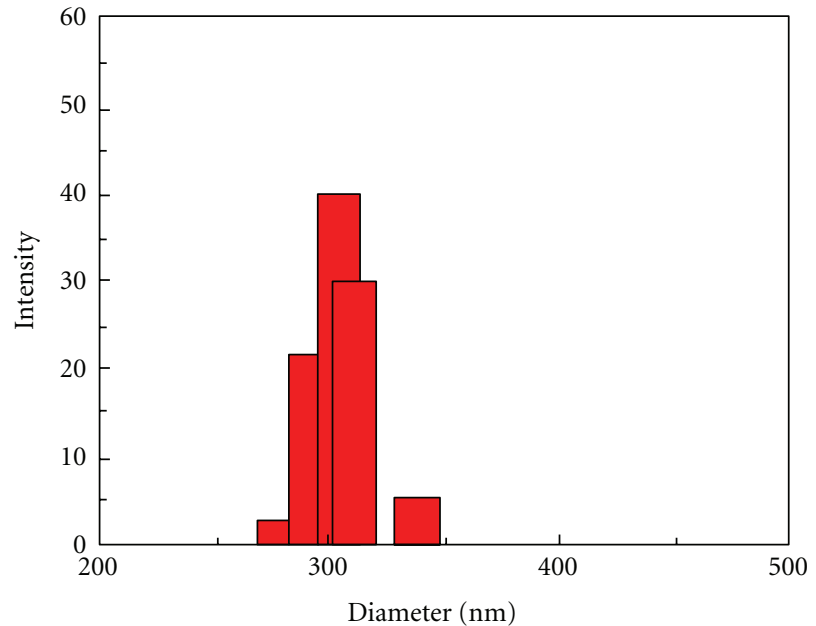

(a)

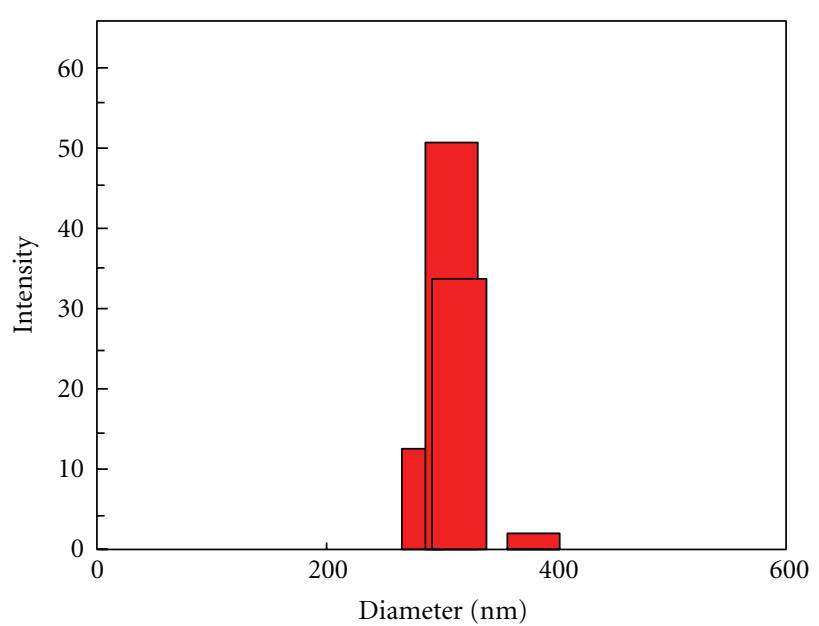

(b)

FIGURE 2: Particle size distribution of calcined double mesoporous core-shell silica nanospheres prepared from silica cores formed at different reaction time of (a) $1 \mathrm{~h}$ and (b) $6 \mathrm{~h}$.

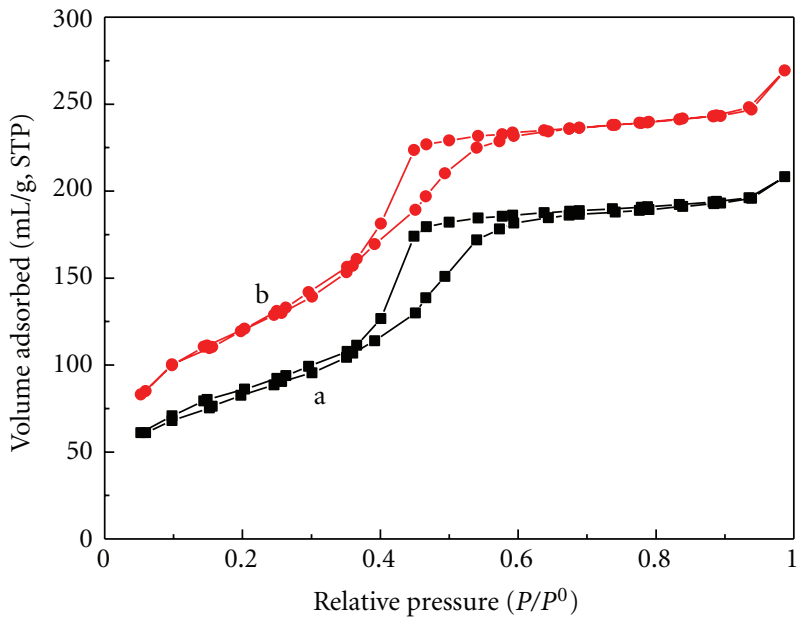

Figure 3: $\mathrm{N}_{2}$ adsorption/desorption isotherms of calcined double mesoporous core-shell silica nanospheres prepared from silica cores formed at different reaction time of (a) $1 \mathrm{~h}$ and (b) $6 \mathrm{~h}$.

for variance homogeneity prior to further statistical analysis. The data were normally distributed and were expressed as the mean \pm standard error of the mean (SEM). Significant differences among groups were analyzed using a oneor two-way ANOVA followed by the Bonferroni multiple comparison test using PRISM statistical analysis software (GraphPad Software). The data were then reanalyzed using a one- or two-way ANOVA followed by Tukey's range test using SPSS software, version 17. Differences were considered to be statistically significant at $P<0.05$.

\section{Results}

3.1. WEV and WEV+NP Inhibit the Growth of MM Cells. Using Silica nanoparticles model, we first investigated the ability of WEV and WEV+NP to induce growth arrest in $\mathrm{MM}$ cells. The effects of WEV and WEV+NP were examined on five patients with MM (Figure 4(a)) and XG2 cell line (Figure 4(b)) at concentrations of $0,1,5,10,25,50,100$, and $1000 \mathrm{ng} / \mathrm{mL}$ and incubation times of $0,1,2,6,12$, 24, 36, and $48 \mathrm{hrs}$ (Figures $4(\mathrm{c})$ and $4(\mathrm{~d})$ ). The resulting cytotoxic effects of $\mathrm{WEV}$ and $\mathrm{WEV}+\mathrm{NP}$ were measured using the MTT uptake method. Data collected from five independent experiments $(n=5)$ demonstrated that WEV and WEV+NP significantly inhibited the growth of MM cells in a dose- and time-dependent manner. The $\mathrm{IC}_{50}$ values for WEV alone and WEV+NP were $25 \mathrm{ng} / \mathrm{mL}$ and $10 \mathrm{ng} / \mathrm{mL}$, respectively. The effect was maximal at $12 \mathrm{~h}$ of incubation. The combination of WEV with NP $(\mathrm{WEV}+\mathrm{NP})$ significantly enhanced the inhibitory effect of WEV in MM cells. The maximal inhibitory effects of WEV and WEV+NP on cell viability were observed $12 \mathrm{~h}$ after treatment with $25 \mathrm{ng} / \mathrm{mL}$ of WEV alone or $10 \mathrm{ng} / \mathrm{mL}$ of WEV+NP. Nevertheless, treatment with NP alone did not affect MM cell viability.

3.2. WEV $+N P$ Inhibits Proliferation of MM Cells. Because the proliferation process is crucial for the maintenance and progression of cancer cells, we monitored the effects of WEV alone or in combination with NP on RPMI-mediated proliferation of MM cells using a CFSE dilution assay followed by flow cytometry. One representative experiment is shown as dot plots in Figure 5. When the MM cells were treated with NP alone, the percentage of proliferating cells was $85 \%$. In contrast, treatment with WEV alone or in combination with NP (WEV+NP) markedly decreased the percentage of proliferating cells to $45 \%$ and $22 \%$, respectively. The total data from the different experiments ( $n=70$ for MM cells from 70 patients) revealed that treatment with WEV alone significantly reduced $(P<0.00)$ the proliferative capacity of MM. Moreover, the combination 


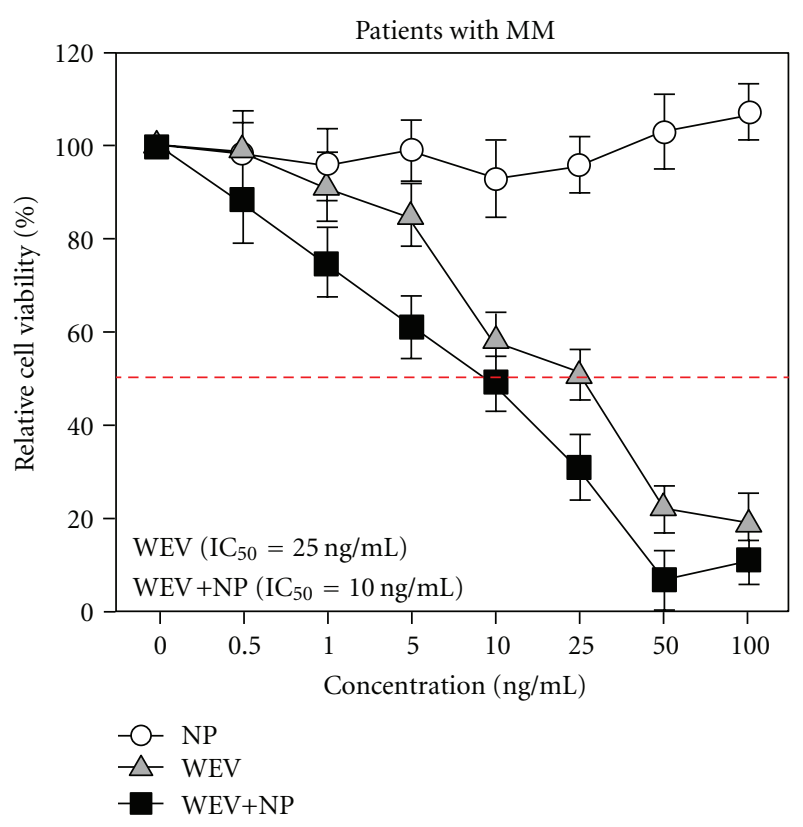

(a)

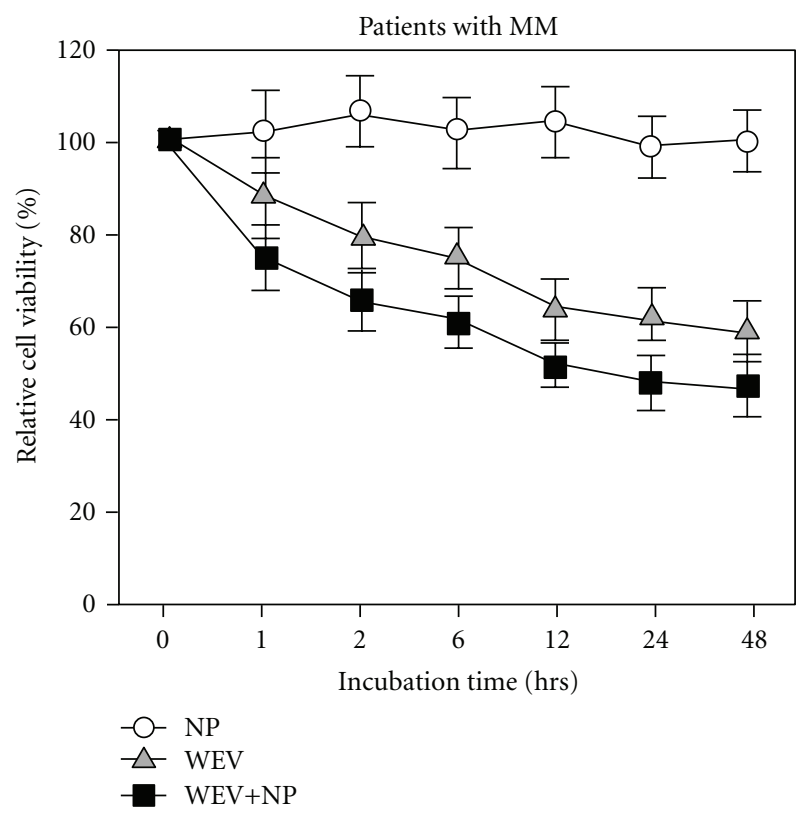

(c)

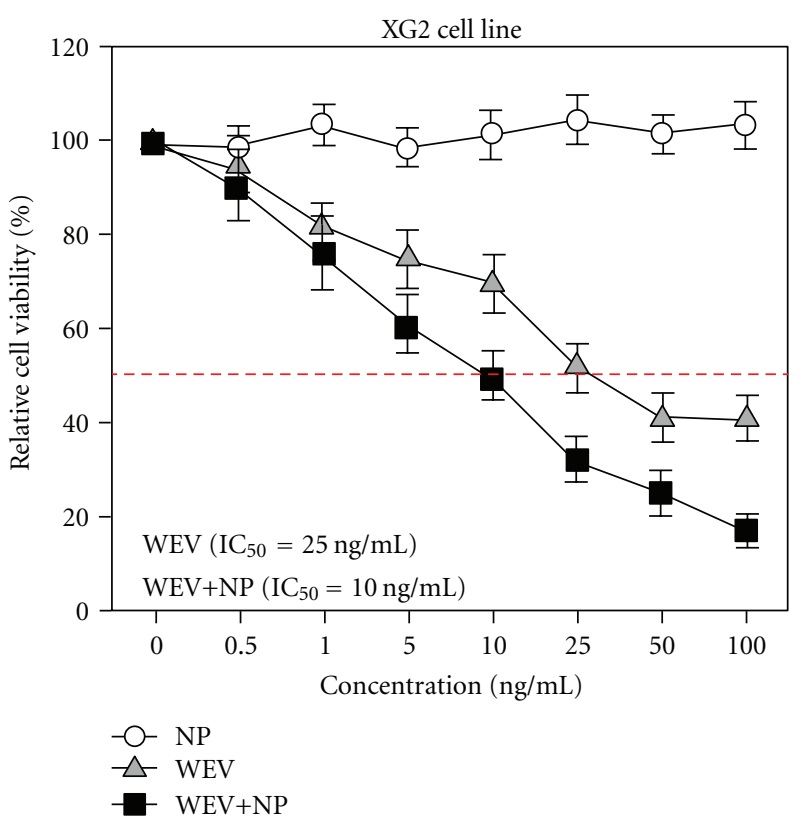

(b)

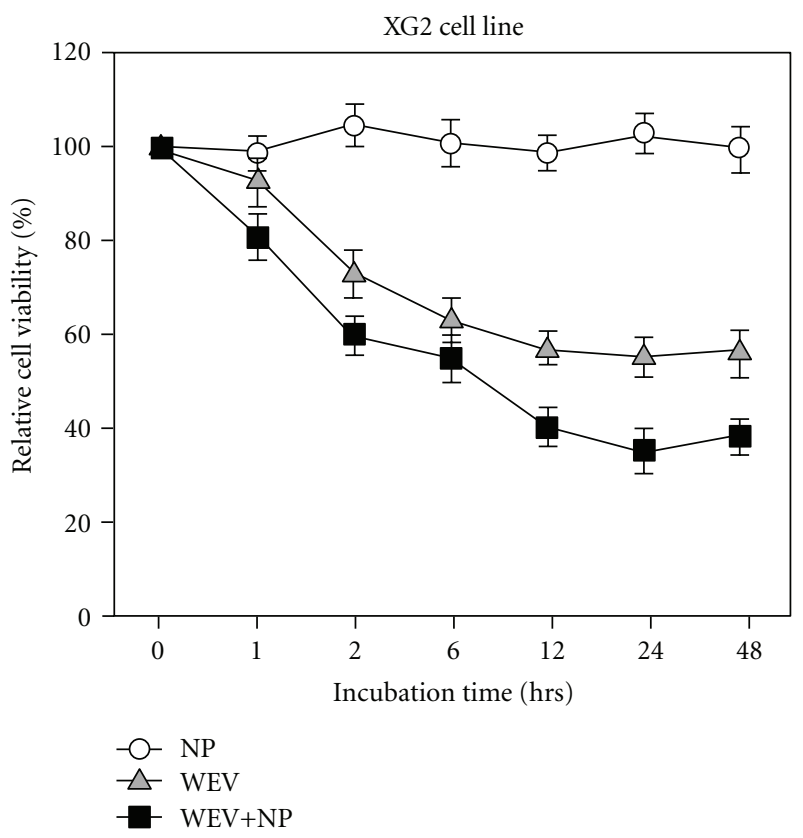

(d)

FigURE 4: Time- and dose-dependent effect of WEV and WEV+NP treatment on cell viability. Cell viability was assessed using the MTT assay. MM cells from patients with MM (a) and XG2 cells (b) were treated overnight with different concentrations $(0,1,5,10,25,50,100$, and $1000 \mathrm{ng} / \mathrm{mL}$ ) of NP (open circles), WEV (gray triangles), or WEV+NP (closed black squares). MM cells from patients with MM (c) and XG2 cells (d) were treated with $25 \mathrm{ng} / \mathrm{mL}$ of NP (open circles), $25 \mathrm{ng} / \mathrm{mL}$ of WEV (gray triangles), or $10 \mathrm{ng} / \mathrm{mL}$ of WEV+NP (closed black squares) for different incubation times $(0,1,2,6,12,24,36$, and $48 \mathrm{hrs})$. The data collected from independent experiments $(n=5)$ are shown, and the results are expressed as the mean percentage of viable cells \pm SEM.

of NP with WEV significantly enhanced the inhibitory effect of WEV on MM, whereas NP alone had no effect (Table 1).

3.3. WEV+NP Induces Apoptosis in MM Cells. The inhibition of cancer cell proliferation, the cessation of cellcycle progression, and the induction of apoptosis have all been targeted in chemotherapeutic strategies for the treatment of cancer. We therefore evaluated whether WEV and WEV+NP alter the cell cycle of MM cells using PI, Annexin V, and flow cytometric analyses. Figure 6 shows one representative experiment; we found that the percentage of apoptotic cells 
TABLE 1: Percentage of proliferating and apoptotic cells, $S$ phase, caspases activities, and oxidative markers among treated and untreated multiple myeloma cells.

\begin{tabular}{|c|c|c|c|c|c|c|c|c|c|}
\hline & \multirow{2}{*}{ Untreated cells } & \multirow{2}{*}{ NP-treated cells } & \multirow{2}{*}{ WEV-treated cells } & \multirow{2}{*}{ WEV+NP-treated cells } & \multicolumn{5}{|c|}{$P$ value } \\
\hline & & & & & A & B & $\mathrm{C}$ & $\mathrm{D}$ & $\mathrm{E}$ \\
\hline $\begin{array}{l}\text { Percentage of } \\
\text { proliferating cells }\end{array}$ & $87.3 \pm 4.7$ & $86.9 \pm 4.5$ & $44.2 \pm 3.5$ & $22.17 \pm 2.1$ & 0.000 & 0.09 & 0.000 & 0.000 & 0.000 \\
\hline $\begin{array}{l}\text { Percentage of } \\
\text { apoptotic cells }\end{array}$ & $3.6 \pm 2.5$ & $4.1 \pm 2.9$ & $84.4 \pm 9.9$ & $90.4 \pm 7.8$ & 0.000 & 0.99 & 0.000 & 0.000 & 0.000 \\
\hline S phase & $60.5 \pm 15.8$ & $59.2 \pm 16.2$ & $30.2 \pm 12.5$ & $23.6 \pm 11.1$ & 0.000 & 0.95 & 0.000 & 0.000 & 0.61 \\
\hline Caspase 3 & $0.8 \pm 0.1$ & $0.9 \pm 0.15$ & $1.9 \pm 0.2$ & $2.7 \pm 0.25$ & 0.000 & 0.96 & 0.000 & 0.000 & 0.001 \\
\hline Caspase 8 & $1.1 \pm 0.1$ & $0.8 \pm 0.1$ & $2.4 \pm 0.3$ & $5.1 \pm 0.45$ & 0.000 & 0.19 & 0.000 & 0.000 & 0.000 \\
\hline Caspase 9 & $1.4 \pm 0.4$ & $1.3 \pm 0.3$ & $3.1 \pm 0.35$ & $6.7 \pm 0.65$ & 0.000 & 0.27 & 0.000 & 0.000 & 0.000 \\
\hline $\mathrm{ROS}(\mathrm{nmol} / \mathrm{mL})$ & $127 \pm 13.7$ & $121 \pm 9$ & $198.5 \pm 18.5$ & $349 \pm 32$ & 0.000 & 0.61 & 0.000 & 0.000 & 0.000 \\
\hline $\begin{array}{l}\text { Hydroperoxide } \\
(\mathrm{mg} / 100 \mathrm{~mL})\end{array}$ & $73 \pm 7.1$ & $75.4 \pm 8.4$ & $134 \pm 12$ & $227 \pm 19$ & 0.000 & 0.73 & 0.000 & 0.000 & 0.000 \\
\hline
\end{tabular}

MM: Multiple myeloma; NP: nanoparticles; WEV: Walterinnesia aegyptia venom; WEV+NP: Walterinnesia aegyptia venom combined with nanoparticles; ROS: reactive oxygen species.

A: $P$ values when different groups are compared.

B: $P$ values when untreatment cells are compared with NP-treated cells.

C: $P$ values when untreatment cells are compared with WEV-treated cells.

D: $P$ values when untreatment cells are compared with WEV+NP-treated cells.

E: $P$ values when WEV-treated cells are compared with WEV+NP-treated cells.

was comparable to $3 \%$ in the untreated cells and $4 \%$ in the NP-treated cells. Treatment with WEV and WEV+NP markedly increased the percentage of apoptotic cells to $90 \%$. This increased induction of apoptosis after treatment with WEV and WEV+NP was inversely correlated with a significant decrease in the percentage of cells in S phase to $25 \%$, respectively, compared to 59\% in both the untreated and NPtreated cells. The total data from different experiments $(n=$ 70 for MM cells from patients) demonstrated that treatment with WEV alone significantly potentiated apoptosis in MM cells (Table 1; $P<0.000$ ). Although NP had no effect on apoptosis induction in MM cells, the combination of NP and WEV significantly increased apoptosis induction compared to WEV alone $(P<0.000)$.

3.4. Caspase Activity. Our results shown in Figure 7 indicated that the level of caspases 3,8 , and 9 activities were increased in MM cells after treatment with WEV and increased more after treatment with WEV+NP. Results showed that there is a significant increase of caspases activity in treated cells in comparison to untreated cells and to treated cells with NP alone (Table 1).

3.5. ROS and Hydroperoxide Levels. Measurements of ROS and Hydroperoxide levels indicated their significant increase in treated MM cells (by WEV and WEV+NP) in comparison to untreated MM cells and to treated cells with NP alone. NP alone did not affect these parameters in MM cells (Table 1).

\section{Discussion}

In this study, we investigated the impact of snake venom, either alone or in combination with silica nanoparticles, on MM cell growth and survival. We used cells isolated from patients who were clinically diagnosed with multiple myeloma. We found that WEV alone and in combination with silica nanoparticles inhibited the growth of MM cells in a dose- and time-dependent manner. Moreover, WEV+NP enhanced the effect of WEV on the cancer cells. The $\mathrm{IC}_{50}$ values of WEV and WEV+NP for the growth inhibition of MM cells were $25 \mathrm{ng} / \mathrm{mL}$ and $10 \mathrm{ng} / \mathrm{mL}$, respectively; these values were the same in MM cells isolated from patients and XG2 cell line, regardless of their sensitivity. However, the sensitivity of cancer cells to WEV and WEV+NP treatment varies because we recently demonstrated that the $\mathrm{IC}_{50}$ values of WEV and WEV+NP that mediated growth inhibition of MCF-7 and MDA-MB-231 breast cancer cells was $50 \mathrm{ng} / \mathrm{mL}$ and $10 \mathrm{ng} / \mathrm{mL}$, respectively $[4,7]$. Furthermore, these $\mathrm{IC}_{50}$ values that mediated growth inhibition of breast cancer cells did not affect the viability of nontumorigenic normal breast epithelial cells (MCF-10) and human normal PBMCs. In addition, it has been previously reported that snake venom can induce apoptosis in many cancer cell lines $[8,9]$.

Uncontrolled proliferation is significant in cell turnover and tumorigenesis. Therefore, we monitored the effects of WEV alone or in combination with NP on the RPMImediated proliferation of MM cells using a CFSE dilution assay followed by flow cytometric analysis. Our data revealed that WEV alone and in combination with NP inhibited the proliferation of MM cells. Therefore, the induction of apoptosis in MM cells may lead to their regression and improved disease prognosis [10]. Thus, agents that are able to induce apoptosis may be useful chemotherapeutic agents against MM.

Although the landscape of MM treatment is rapidly changing, this disease is largely incurable [11]. Several chemotherapeutic agents (e.g., vincristine, dexamethasone, 

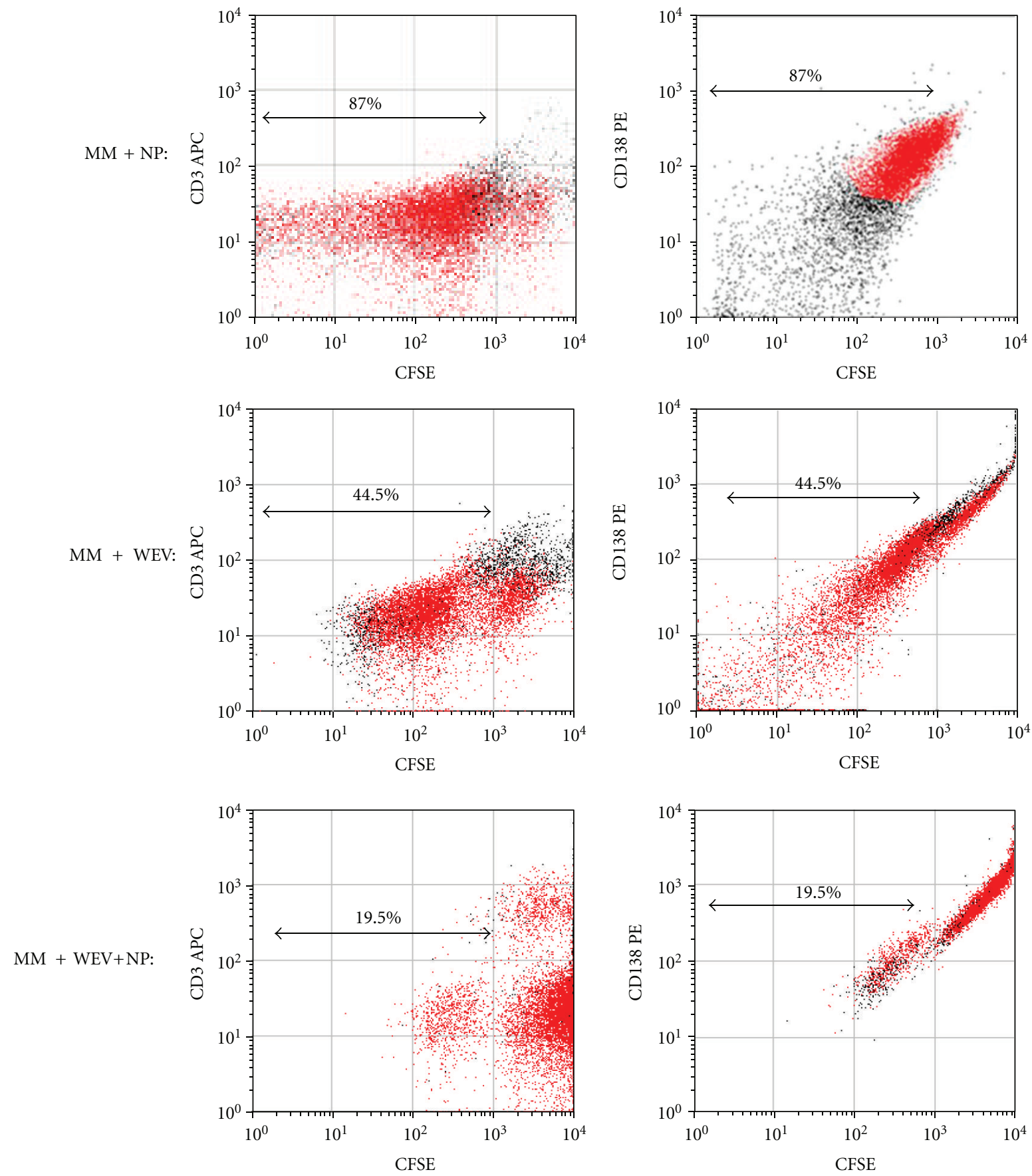

FIGURE 5: WEV and WEV+NP dampen the proliferation of MM cells. The ability of MM cells to proliferate spontaneously was evaluated after treatment with NP, WEV, and WEV+NP using CFSE assays and flow cytometry. A representative experiment showing the analysis of CFSE staining in MM cells from one patient after gating on the viable cells.

and melphalan) are currently used to treat MM. However, these drugs have the disadvantage of increasing the risk of developing secondary hematologic malignancies, such as therapy-related myelodysplastic syndromes [12]. Therefore, there is a crucial need to further identify biological factors and mechanisms that are responsible for MM cell survival, tumorigenesis, and drug resistance [13].

Generally, chemotherapeutic drugs attack on both normal and tumor cells nonspecifically causing life-threatening side effects, necessitating targeted drug delivery to tumors [14]. Indeed, the therapeutic molecule must generally: cross one or various biological membranes before diffusing through the plasma membrane to finally gain access to the appropriate organelle, where the biological target is located. For those drugs whose target is located intracellularly, deviating from this ideal path may not only decrease the drug efficiency, but also entail side effects and toxicity. For these reasons, more than 30 years ago, the idea emerged to 


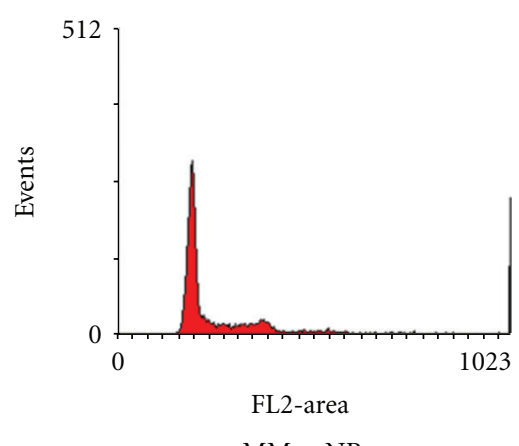

$\mathrm{MM}+\mathrm{NP}$

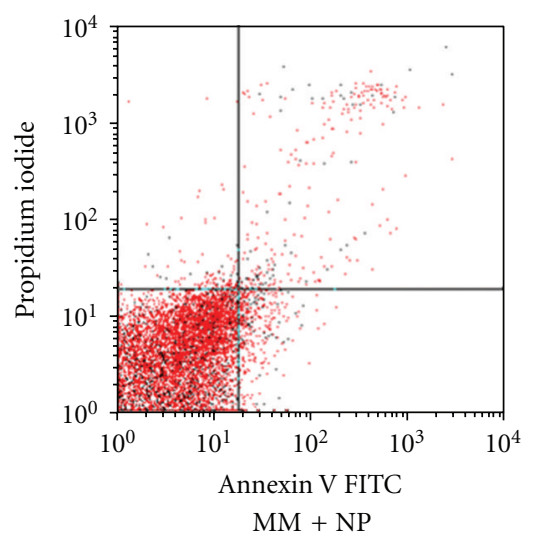

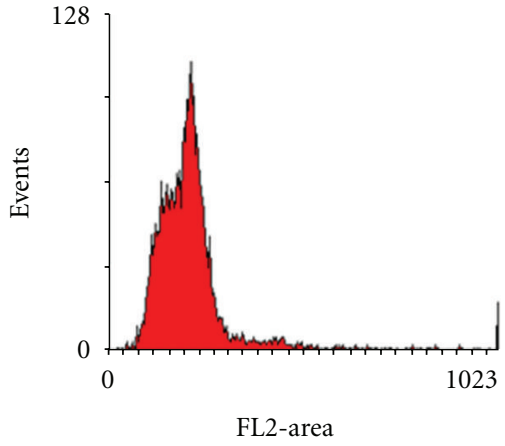

$\mathrm{MM}+\mathrm{WEV}$

(a)

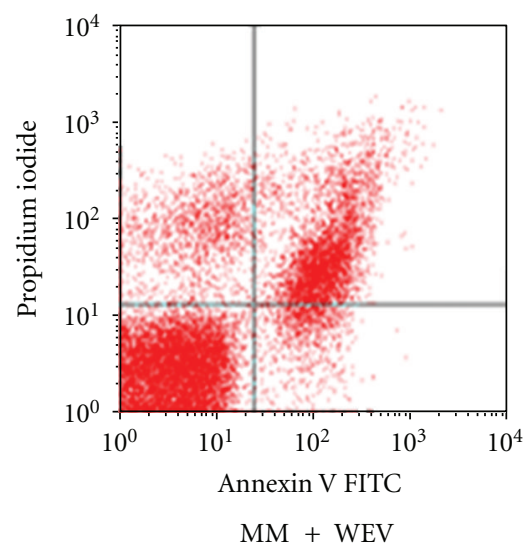

(b)

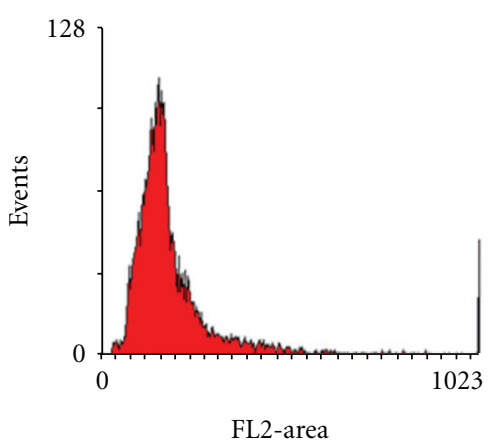

$\mathrm{MM}+\mathrm{WEV}+\mathrm{NP}$

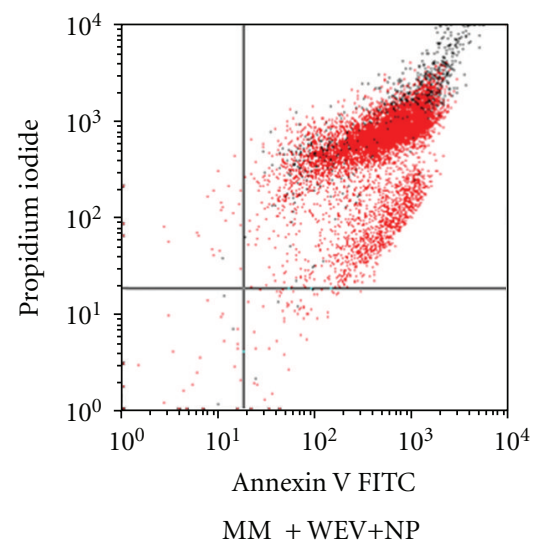

FIGURE 6: The impact of WEV+NP on the cell cycle and apoptosis in MM cells. (a) The ability of WEV and WEV+NP to alter the cell cycle of MM cells was evaluated using propidium iodide (PI) and flow cytometry. PI-labeled cells were gated depending on the PI-area and PI-width to calculate the G1, S, G2/M and sub-G1 (apoptotic cells) cellcycle phases. Histograms of PI-stained cells from one representative experiment are shown. (b) Dot plot of annexin V FITC versus PI showing increase apoptosis percentage on MM cells evaluated after treatment with $\mathrm{WEV}+\mathrm{NP}$.

tailor carriers small enough to ferry the active substance to the target cell and its relevant subcellular compartment [15]. Various types of nanoparticles, such as liposomes, polymeric micelles, dendrimers, superparamagnetic iron oxide crystals, and colloidal gold, have been employed in targeted therapies for cancer [ 16,17$]$. Nanocarriers offer unique possibilities to overcome cellular barriers in order to improve the delivery of various drug candidates [15]. Recent studies showed an efficient tumor targeting by nanoparticles through the enhanced permeability and retention effect [18-20]. Delivery of drug-loaded nanoparticles has achieved success in advanced thyroid cancer [21] and breast carcinoma.

Here, while venom-free nanoparticles had no effect on MM cells, the combination of WEV with nanoparticles increased the efficiency of WEV to fight MM cells by twofold when compared to WEV alone. Despite the large interest of using nanoparticles in biomedical applications, a clear understanding of their cellular uptake and transport is still lacking. They appear to translocate across cells via clathrinand macropinocytosis-mediated endocytosis [22]. Experimental data agreed with that, whereas PLL-g-PEG-DNA nanoparticles were internalized in an energy-dependent manner after $2 \mathrm{~h}$ and accumulated in the perinuclear region after more than $6 \mathrm{~h} \mathrm{[23].} \mathrm{In} \mathrm{which} \mathrm{the} \mathrm{authors} \mathrm{demonstrated}$ that the nanoparticles were found to be stable within the cytoplasm for at least $24 \mathrm{~h}$ and did not colocalize with the endosomal pathway. Furthermore, nanoparticle uptake was approximately $50 \%$ inhibited by genistein, an inhibitor of the caveolae-mediated pathway. However, clathrin-mediated endocytosis and macropinocytosis pathways were reduced by $17 \%$ and $24 \%$, respectively, in the presence of the respective inhibitors. These findings suggest that PLL-g-PEG-DNA nanoparticles enter by several pathways and might therefore be an efficient and a versatile tool to deliver therapeutic DNA [23]. On the other hand, some authors demonstrated that silica nanoparticles induced cells apoptosis (via ROS) [24]. For that, we studied the ROS and hydroperoxide in NPtreated cells, and we demonstrated no effect on them. This discrepancy may be due larger dose used by the previous authors.

The release of loaded drugs from nanoparticles may be controlled in response to changes in environmental conditions such as temperature and $\mathrm{pH}$. Biodistribution profiles and anticancer efficacy of nanodrugs in vivo would 


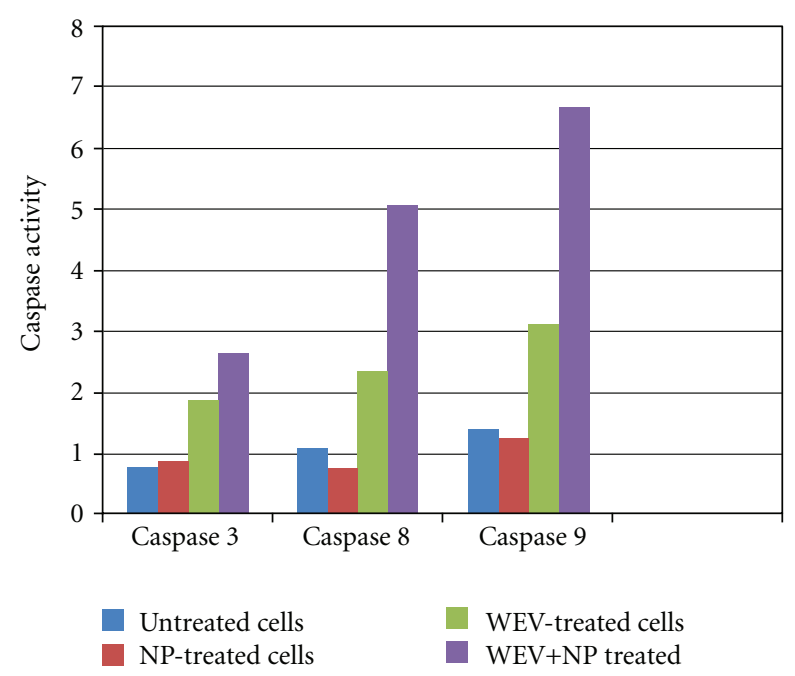

Figure 7: The caspases (3,8 and 9) activity among treated and untreated multiple myeloma cells.

be different depending upon their size, surface charge, PEGylation, and other biophysical properties [16]. Through the induction of T-cell immunity, nanoparticles may not only provide a therapeutic approaches for cancer, but also provide a new prophylactic strategy for infectious disease [25], the reason why nanotechnology is expected to play a vital role in the rapidly developing field of nanomedicine, creating innovative solutions and therapies for currently untreatable diseases and providing new tools for various biomedical applications, such as drug delivery and gene therapy [26].

\section{Conclusions}

This study revealed the unique biological effects of WEV and WEV+NP on MM cells that enable these compounds to function as treatments for MM.

$\begin{array}{ll}\text { Abbreviations } \\ \text { MM: } & \text { Multiple myeloma } \\ \text { NP: } & \text { Nanoparticles } \\ \text { WEV: } & \text { Walterinnesia aegyptia venom } \\ \text { WEV+NP: } & \text { Walterinnesia aegyptia venom combined } \\ & \text { with nanoparticles. }\end{array}$

\section{Conflict of Interests}

All authors have read and agreed the contents of the paper and approved the submission. The authors declare that they have no conflict of interests, state that the manuscript has not been published or submitted elsewhere, state that the work complies with the ethical policies of the journals and state that the work has been conducted under internationally accepted ethical standards after relevant ethical review.

\section{Authors' Contribution}

D. Sayed carried out the experiments, performed the statistical analysis, prepared the figures, and drafted the paper. M. K. Al-Sadoon was responsible for the extraction of snake venom and participated in the manuscript revision. G. Badr formulated the study design and participated in the manuscript revision.

\section{Acknowledgments}

This work was supported by the National Plan for Science and Technology (NPST) funded by the King Abdulaziz City for Science and Technology (KACST) through Project no. 10-BIO969-02. The authors also acknowledge Dr. Ahmed El-Toni at the King Abdullah Institute for Nanotechnology, King Saud University, for loading venom onto the silica nanoparticles.

\section{References}

[1] D. Gupta, T. Hideshima, and K. C. Anderson, "Novel biologically based therapeutic strategies in myeloma," Reviews in Clinical and Experimental Hematology, vol. 6, no. 3, pp. 301324, 2002.

[2] M. Zhao, J. Ma, H.-Y. Zhu et al., "Apoptosis in human multiple myeloma cells through targeting the trinity of CK2, Cdc37 and Hsp90," Molecular Cancer, vol. 10, article 104, 2011.

[3] F. S. Markland, K. Shieh, Q. Zhou et al., "A novel snake venom disintegrin that inhibits human ovarian cancer dissemination and angiogenesis in an orthotopic nude mouse model," Haemostasis, vol. 31, no. 3-6, pp. 183-191, 2001.

[4] G. Badr, M. K. Al-Sadoon, A. M. El-Toni, and M. Daghestani, "Walterinnesia aegyptia venom combined with silica nanoparticles enhances the functioning of normal lymphocytes through PI3K/AKT, NFkB and ERK signaling," Lipids in Health and Disease, vol. 11, article 27, 2012.

[5] D. J. Son, M. H. Park, S. J. Chae et al., "Inhibitory effect of snake venom toxin from Vipera lebetina turanica on hormonerefractory human prostate cancer cell growth: induction of apoptosis through inactivation of nuclear factor $\kappa \mathrm{B}$," Molecular Cancer Therapeutics, vol. 6, no. 2, pp. 675-683, 2007.

[6] G. Barratt, "Colloidal drug carriers: achievements and perspectives," Cellular and Molecular Life Sciences, vol. 60, no. 1, pp. 21-37, 2003.

[7] M. K. Al-Sadoon, M. A. Abdel-Maksoud, and D. M. Rabah, "Badr G. Induction of apoptosis and growth arrest in human breast carcinoma cells by a snake (Walterinnesia aegyptia) venom combined with silica nanoparticles: crosstalk between Bcl2 and caspase 3," Cellular Physiology and Biochemistry, vol. 30, no. 3, pp. 653-665, 2012.

[8] M. H. Park, M. R. Jo, D. Won et al., "Snake venom toxin from Vipera lebetina turanica induces apoptosis in colon cancer cells via upregulation of ROS- and JNK-mediated death receptor expression," BMC Cancer, vol. 12, no. 12, article 228, 2012.

[9] J. K. Song, M. R. Jo, M. H. Park et al., "Cell growth inhibition and induction of apoptosis by snake venom toxin in ovarian cancer cell via inactivation of nuclear factor $\kappa \mathrm{B}$ and signal transducer and activator of transcription 3," Archives of Pharmacal Research, vol. 35, no. 5, pp. 867-876, 2012. 
[10] E. M. Bruckheimer and N. Kyprianou, "Apoptosis in prostate carcinogenesis: a growth regulator and a therapeutic target," Cell and Tissue Research, vol. 301, no. 1, pp. 153-162, 2000.

[11] T. Nishihori and M. Alsina, "Advances in the autologous and allogeneic transplantation strategies for multiple myeloma," Cancer Control, vol. 18, pp. 258-267, 2011.

[12] Y. Ishii, H. H. Hsiao, G. Sashida et al., "Derivative $(1 ; 7)(\mathrm{q} 10$; p10) in multiple myeloma. A sign of therapy-related hidden myelodysplastic syndrome," Cancer Genetics and Cytogenetics, vol. 167, no. 2, pp. 131-137, 2006.

[13] G. Badr, E. A. Lefevre, and M. Mohany, "Thymoquinone inhibits the CXCL12-induced chemotaxis of multiple myeloma cells and increases their susceptibility to Fasmediated apoptosis," PLOS ONE, vol. 6, no. 9, Article ID e23741, 2011.

[14] L. P. Kolluru, S. A. Rizvi, M. D'Souza, and M. J. D'Souza, "Formulation development of albumin based theragnostic nanoparticles as a potential delivery system for tumor targeting," Journal of Drug Targeting. In press.

[15] H. Hillaireau and P. Couvreur, "Nanocarriers' entry into the cell: relevance to drug delivery," Cellular and Molecular Life Sciences, vol. 66, no. 17, pp. 2873-2896, 2009.

[16] J. Wang, M. Sui, and W. Fan, "Nanoparticles for tumor targeted therapies and their pharmacokinetics," Current Drug Metabolism, vol. 11, no. 2, pp. 129-141, 2010.

[17] F. Haque, D. Shu, Y. Shu et al., "Ultrastable synergistic tetravalent RNA nanoparticles for targeting to cancers," Nano Today, vol. 7, pp. 245-257, 2012.

[18] H. Y. Nam, S. M. Kwon, H. Chung et al., "Cellular uptake mechanism and intracellular fate of hydrophobically modified glycol chitosan nanoparticles," Journal of Controlled Release, vol. 135, no. 3, pp. 259-267, 2009.

[19] H. Koo, M. S. Huh, I. C. Sun et al., "In vivo targeted delivery of nanoparticles for theranosis," Accounts of Chemical Research, vol. 44, pp. 1018-1028, 2011.

[20] S. M. Lim, T. H. Kim, H. H. Jiang et al., "Improved biological half-life and anti-tumor activity of TNF-related apoptosisinducing ligand (TRAIL) using PEG-exposed nanoparticles," Biomaterials, vol. 32, no. 13, pp. 3538-3546, 2011.

[21] B. Koppolu, Z. Bhavsar, A. S. Wadajkar et al., "Temperaturesensitive polymer-coated magnetic nanoparticles as a potential drug delivery system for targeted therapy of thyroid cancer," Journal of Biomedical Nanotechnology, vol. 8, pp. 983-990, 2012.

[22] X. Zeng, Y. Zhang, and A. M. Nyström, "Endocytic uptake and intracellular trafficking of bis-MPA based hyperbranched copolymer micelles in breast cancer cells," Biomacromolecules, vol. 13, no. 11, pp. 3814-3822, 2012.

[23] T. Lühmann, M. Rimann, A. G. Bittermann, and H. Hall, "Cellular uptake and intracellular pathways of PLL-g-PEGDNA nanoparticles," Bioconjugate Chemistry, vol. 19, no. 9, pp. 1907-1916, 2008.

[24] J. Ahmad, M. Ahamed, M. J. Akhtar et al., "Apoptosis induction by silica nanoparticles mediated through reactive oxygen species in human liver cell line HepG2," Toxicology and Applied Pharmacology, vol. 259, no. 2, pp. 160-168, 2012.

[25] T. Kozako, N. Arima, M. Yoshimitsu, S. I. Honda, and S. Soeda, "Liposomes and nanotechnology in drug development: focus on oncotargets," International Journal of Nanomedicine, vol. 7, pp. 4943-4951, 2012.

[26] T. Dos Santos, J. Varela, I. Lynch, A. Salvati, and K. A. Dawson, "Effects of transport inhibitors on the cellular uptake of carboxylated polystyrene nanoparticles in different cell lines," PLoS ONE, vol. 6, Article ID e24438, 2011. 


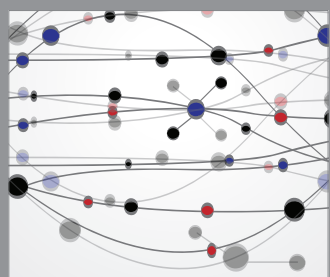

The Scientific World Journal
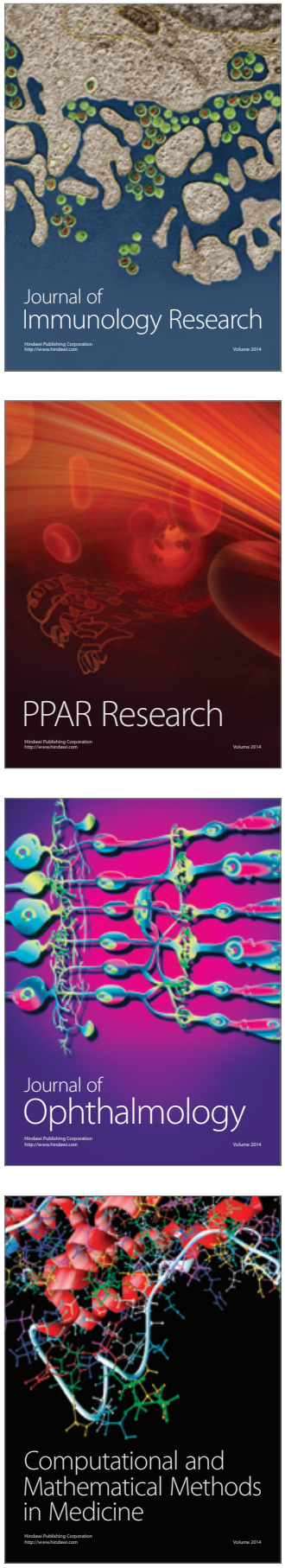

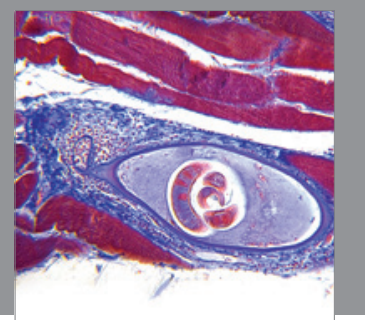

Gastroenterology

Research and Practice
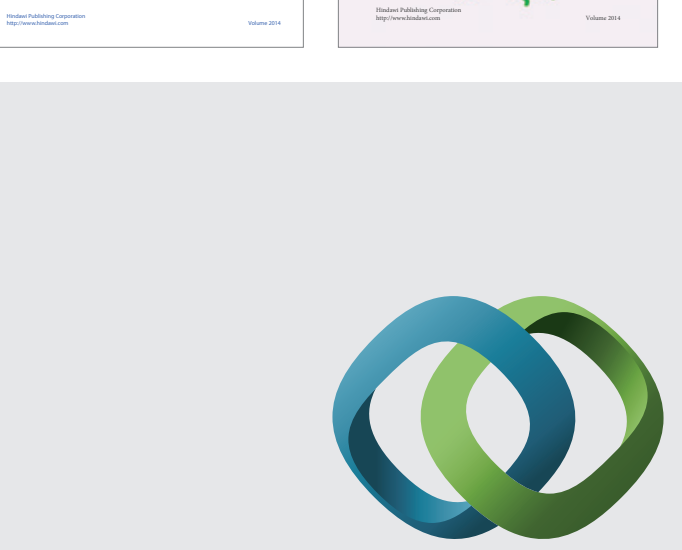

\section{Hindawi}

Submit your manuscripts at

http://www.hindawi.com
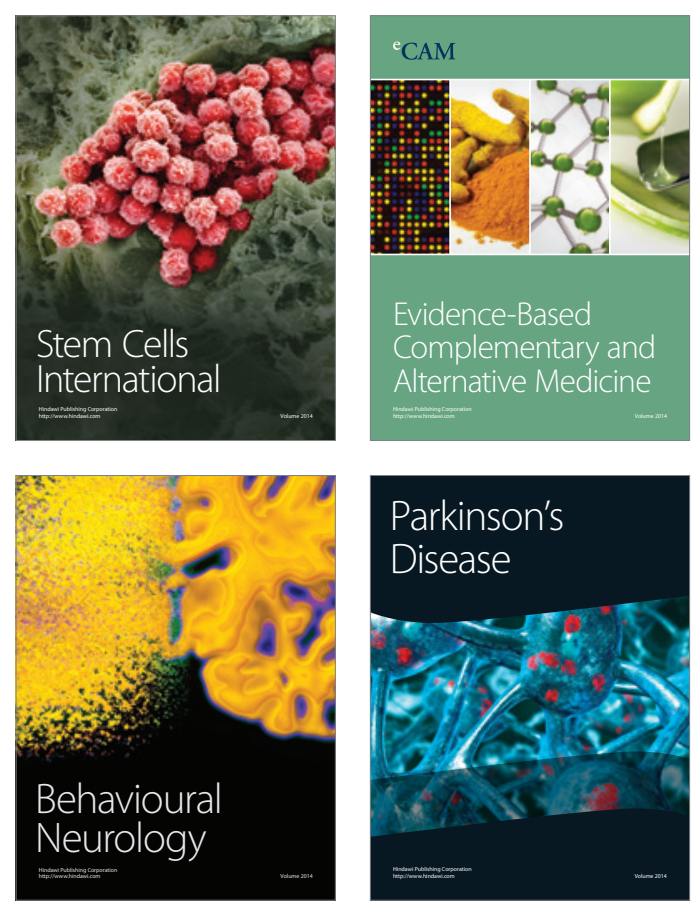

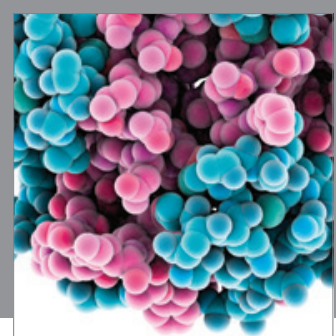

Journal of
Diabetes Research

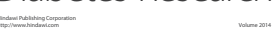

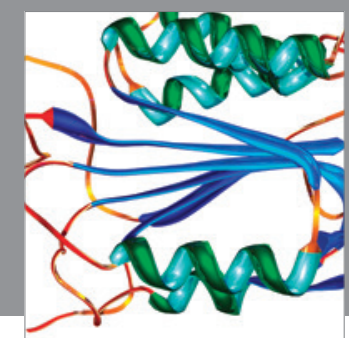

Disease Markers
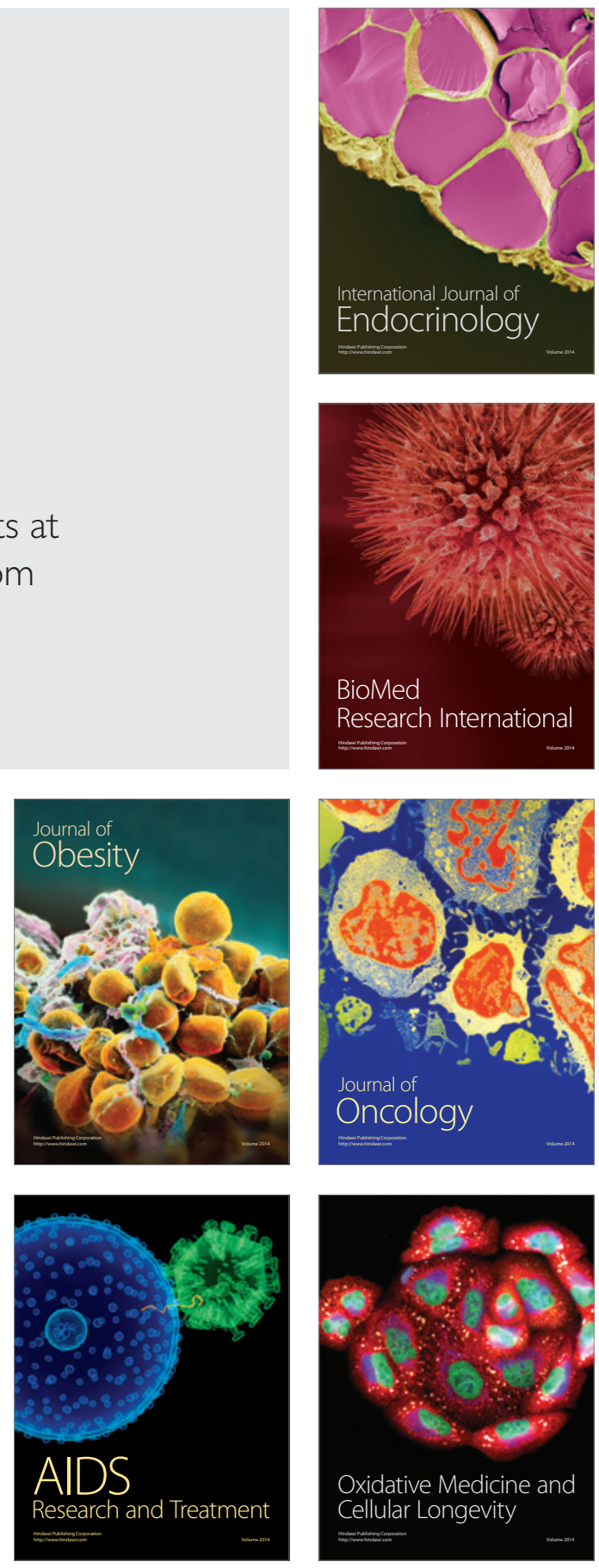